\title{
All-electronic suppression of mode hopping noise in diode lasers
}

Bager, L.

Published in:

I E E E Photonics Technology Letters

Link to article, DOI:

$10.1109 / 68.62023$

Publication date:

1990

Document Version

Publisher's PDF, also known as Version of record

Link back to DTU Orbit

Citation (APA):

Bager, L. (1990). All-electronic suppression of mode hopping noise in diode lasers. I E E E Photonics Technology Letters, 2(12), 899-901. https://doi.org/10.1109/68.62023

\section{General rights}

Copyright and moral rights for the publications made accessible in the public portal are retained by the authors and/or other copyright owners and it is a condition of accessing publications that users recognise and abide by the legal requirements associated with these rights.

- Users may download and print one copy of any publication from the public portal for the purpose of private study or research.

- You may not further distribute the material or use it for any profit-making activity or commercial gain

- You may freely distribute the URL identifying the publication in the public portal

If you believe that this document breaches copyright please contact us providing details, and we will remove access to the work immediately and investigate your claim. 


\title{
All-Electronic Suppression of Mode Hopping Noise in Diode Lasers
}

\author{
L. BAGER
}

\begin{abstract}
We present a simple all-electronic stabilization scheme for suppression of external cavity mode hopping noise in diode lasers. This excess noise is generated when the laser is subjected to optical feedback and may degrade the overall performance of optical systems including sensors. Suppression of the noise has been demonstrated to improve the sensitivity of a coherent sensor by more than $23 \mathrm{~dB}$.
\end{abstract}

\section{INTRODUCTION}

$\mathrm{F}^{\mathrm{o}}$ OR many optical sensor configurations a single-mode laser source has an advantage over multimode and incoherent light sources with respect to coherence length and intrinsic noise properties. Single-mode operation can be ensured by DFB lasers with optical isolators. However, these components are still very expensive and therefore not yet applicable to for example high volume, low cost fiber optic sensors.

An electronic stabilization scheme which can ensure single-mode operation of CD-type laser diodes is investigated in this letter. An electronic stabilization scheme has the advantage of being potentially inexpensive. Especially the ability of the stabilization scheme to compensate for the influence of optical feedback is investigated.

Optical feedback (OFB) has been treated extensively in the literature [1], [2]. The OFB has a significant effect on laser intensity and frequency noise. One reason for the interest in OFB has been to adopt the feedback as a linewidth narrowing effect compared to the linewidth of the solitary single mode laser. Recently diode lasers with an on-chip external cavity generating the OFB have reached a commercial stage (Sharp LT080).

For many sensor applications, however, unwanted OFB effects such as an increased intensity noise level may degrade the overall performance of the sensor.

Optical [3] and electronic [4] noise reduction schemes have been proposed to improve the performance of the sensor. A nonoptical control loop to eliminate the external cavity mode hopping has been reported [4]. The scheme utilizes a fixed noise level as the regulating reference. The reference, however, has to be above the minimum noise level of the laser.

Here we present a simple all electronic stabilization scheme that forces the laser to operate at the noise minimum. The scheme is designed for suppression of the mode hopping noise in sensor configurations.

In the next section we briefly summarize the important parameters which characterize the optical feedback. Next the

Manuscript received July 23, 1990. This work was supported by the Danish Technical Research Council.

The author is with the Center for Broadband Telecommunications, Electromagnetics Institute, Technical University of Denmark, DK 2800 Lyngby, Denmark.

IEEE Log Number 9040757. electronic stabilizing principle is described in detail followed by experiments and discussion.

\section{BACKGROUND}

When a semiconductor laser is exposed to optical feedback, a compound cavity is formed. The compound cavity may consist of several reflecting surfaces for example the reference and the object in a Michelson interferometer. Here we concentrate on the case with only one reflection. An important parameter to describe the influence of OFB is the feedback coefficient $\kappa$ given by [5]

$$
\kappa=\frac{1}{\tau_{d}} \frac{1-R}{\sqrt{ } R} \sqrt{ } f_{\mathrm{ext}}
$$

where $\tau_{d}$ is the roundtrip time in the laser cavity and $R$ is the power reflectivity of the laser facets. $f_{\text {ext }}$ is the feedback ratio between the reflected power to the laser cavity and the emitted power.

The phase condition in the case of small external reflections, i.e, $f_{\mathrm{ext}} \ll R$, is dependent on the feedback coefficient $\kappa[5]$ :

$$
\begin{gathered}
\omega_{0} \tau-\omega \tau=C \sin (\omega \tau+\operatorname{Arctan} \alpha) \\
C=\kappa \tau \sqrt{ } 1+\alpha^{2} .
\end{gathered}
$$

$C$ is the feedback parameter, $\omega_{0}$ the solitary laser angular velocity, $\tau$ the roundtrip time in the external cavity, and $\alpha$ the linewidth enhancement factor [6].

For low levels of feedback $C<1$ there is only one stable solution to (2a). Therefore the laser wavelength can be tuned continuously with the feedback phase $\omega_{0} \tau$. However, when $C$ exceeds unity there are two stable solutions for certain values of the phase $\omega_{0} \tau$ [7]. Experimentally, the phase limit $(C=1)$ is identified as the onset of a splitting of the oscillating mode when the two stable solutions are present [1]. When the feedback phase $\omega_{0} \tau$ is changed, the laser may perform mode hopping between two stable solutions at a switching time $\Delta t$ depending on the feedback level and the phase [2]. An analytical treatment of the solution to (2) is given in [7].

The mode hopping $(\mathrm{MH})$ which occurs when $C$ exceeds unity is between two stable solutions with a slightly different excess gain [2]. This gain difference accounts for a small difference in the intensity of the two modes and thereby the MH generates intensity noise. This excess noise is used as input to the stabilizing circuit.

\section{Stabilization Principle}

The all electronic stabilization principle utilizes a low amplitude, low frequency modulation of the laser. The modulation results in a wavelength swing which is used to predict the mode hopping situations. Phase sensitive detection of the 


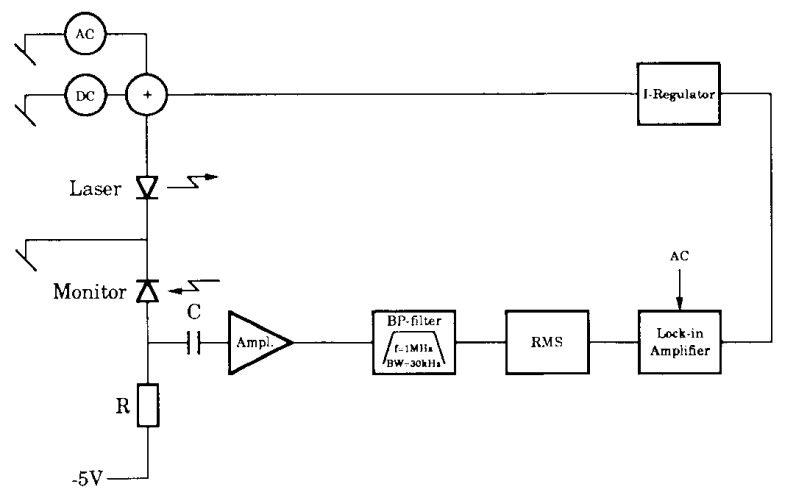

Fig. 1. The all-electronic stabilization scheme. The intensity noise level, amplified and bandpass filtered at $1 \mathrm{MHz}$, is compared to the modulation waveform in a lock-in-amplifier. The amplifier output is the regulating error signal fed back to the laser via an integrating regulator.

noise level with the modulating waveform as a reference provides a feedback signal for stabilization. The realized circuit is shown in Fig. 1.

The intensity noise is measured with the built-in monitor diode in the laser package. The rms level in a $30 \mathrm{kHz}$ bandwidth at a centre frequency of approximately $1 \mathrm{MHz}$ is the error signal that is compared to the ac bias waveform in a lock-in amplifier (LIA). The output of the LIA is then fed back to the laser through an integrating regulator. The wavelength swing due to the ac signal has to be small compared to the external cavity mode spacing not to produce two mode transitions in one modulation period.

The FM bias of the laser predicts the $\mathrm{MH}$ events by modulating the OFB phase. An example of a bias waveform together with the monitor signal is shown in Fig. 2. An increased noise level in the positive half periods of the bias waveform is observed. The noise level increases when the OFB phase is driving the laser towards a $\mathrm{MH}$ event. Thereby it is possible to predict the $\mathrm{MH}$ events. The stabilizing scheme can prevent the laser from performing $\mathrm{MH}$ in large ranges of operating currents and temperatures as well as OFB levels. The phase difference between the laser drive current and the monitor signal is due to an integrated power control circuit used for this measurement only.

\section{EXPERIMENT}

A Fabry-Perot laser operating at $782 \mathrm{~nm}$ wavelength is subject to optical feedback from a plane mirror forming a 27 $\mathrm{cm}$ long external cavity. The OFB level is controlled by a neutral density filter. The wavelength is monitored simultaneously by a scanning Fabry-Perot analyzer with a free spectral range of $7.5 \mathrm{GHz}$ and by a monochromator as shown in Fig. 3. The laser is modulated with a $3.5 \mu \mathrm{A}$ (rms) amplitude at $1 \mathrm{kHz}$ to provide the probe signal.

To simulate effects of temperature perturbations, the laser is mounted on a Peltier cooler. The current signal for this element is supplied by a PID-regulator with two inputs. One input is used for a PT-100 temperature sensor to establish a fixed temperature set point of $25^{\circ} \mathrm{C}$. An auxiliary input is utilized for generating a small $\left(< \pm 0.2^{\circ} \mathrm{C}\right)$ sinusoidal perturbation of the set point with a frequency of $10^{-2} \mathrm{~Hz}$.

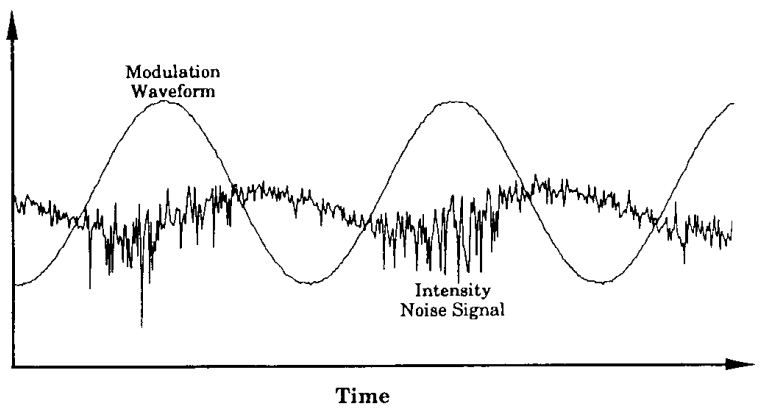

Fig. 2. The ac modulation waveform is here shown quantitatively with the corresponding intensity noise signal. The measurement is performed when the laser is close to a mode hopping situation. Notice the increased noise level in the positive modulation half period.

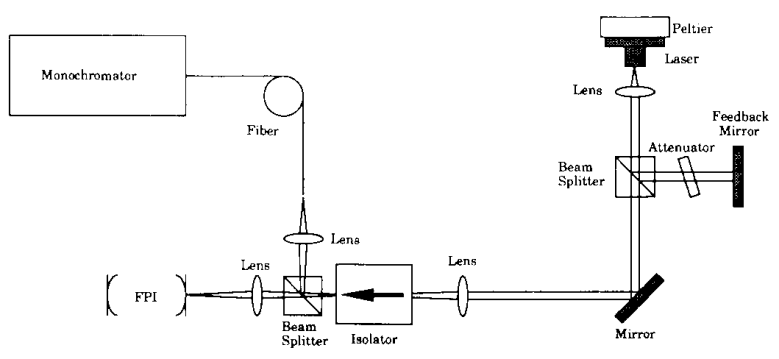

Fig. 3. The optical setup. The laser is mounted on a Peltier cooler for controlling the temperature of the laser chip. The feedback level is set using the plane mirror and a neutral density filter, slightly tilted to avoid reflections back to the laser cavity from the filter surface. The laser wavelength is monitored on a Fabry-Perot interferometer (FPI) and a monochromator, respectively.

\section{RESUlts}

For the laser used in our experiments, the frequency drift with temperature is $9 \mathrm{GHz} /{ }^{\circ} \mathrm{C}$. The external cavity mode spacing is $0.56 \mathrm{GHz}$ so the temperature perturbation generates about 12 mode transitions in each period of $100 \mathrm{~s}$. In other words, two consecutive mode transitions can be distinguished clearly. The phase condition limit $(C=1)$ was tified by adjusting the neutral density filter in order to determine the coupling efficiency of the optical feedback. Using $\alpha=6, \tau_{d}=8 \mathrm{ps}$ and $R=0.32$ in (1) and (2), the calibration of the OFB level can be performed. The phase condition limit could be identified using the Fabry-Perot analyzer to show the onset of the splitting of the single mode $(C<1)$ into two stable modes $(C>1)$ for certain values of the feedback phase. This limit occurred at a feedback level of $-70.5 \mathrm{~dB}$, comparing well to results obtained for a DFB laser [1].

When used for the temperature perturbed laser source shown in Fig. 3, the stabilization circuit shown in Fig. 1 suppresses the induced $\mathrm{MH}$ and thereby leads to a considerable noise reduction. An example of the wavelength drift as well as the intensity noise reduction is shown in Fig. 4. The stabilization scheme turns the frequency drift with temperature into a frequency jitter with a deviation of less than 65 $\mathrm{MHz}$. The intensity noise reduction in Fig. 4(c) is more than $25 \mathrm{~dB}$ when the temperature is changed. The two measurements are performed under similar conditions but with and 


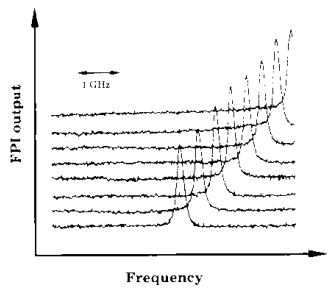

(a)

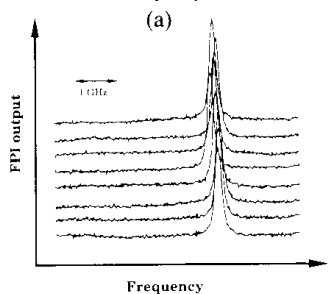

(b)
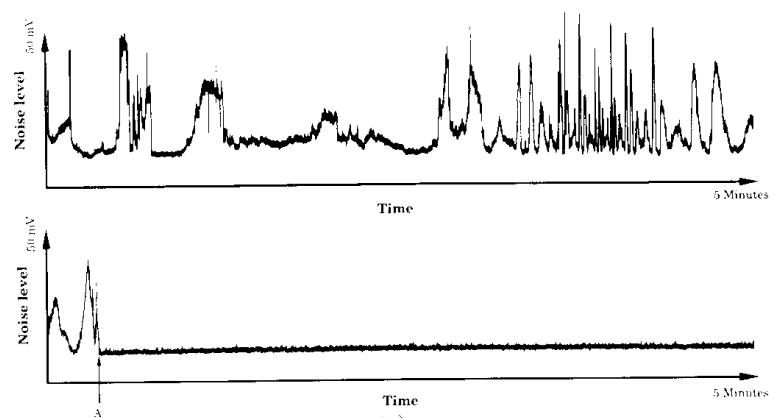

(c)

Fig. 4. (a), (b) The output of the Fabry-Perot Interferometer when the temperature of the laser is slightly changed $\left(<1{ }^{\circ} \mathrm{C}\right)$. (a) without and (b) with stabilization, respectively. The eight scans in (a) and (b) are vertically displaced for clarity. (c) The intensity noise level of the unstabilized laser, measured at $1 \mathrm{MHz}$ in a $30 \mathrm{kHz}$ bandwidth (upper curve). In the lower curve the stabilizing circuit is switched on at $A$. Notice that the laser is kept at a noise minimum with the stabilization. In both curves in (c) the temperature of the laser is varied a few degrees.

without the stabilizing circuit switched on, respectively. An integrating regulator tends to run full as the disturbing signal is increased. Our regulation is able to stabilize the MH noise in a temperature range of app. $5^{\circ} \mathrm{C}$. A reduced gain of the error signal of cause can increase this range. The gain reduction will, however, increase the frequency jitter. As a compromise our results are obtained with an error signal gain of $92 \mathrm{~dB}$, including the LIA gain.

The excess noise from the $\mathrm{MH}$ contributes to the overall noise floor in the detector system in a sensor setup. Stabilizing the laser in an amplitude based sensor will improve the sensitivity of the system corresponding to the reduction in the intensity noise $(25 \mathrm{~dB})$.

With the FP analyzer, the frequency noise related to a mode transition is measured. The noise reduction when $\mathrm{MH}$ is suppressed is $15-20 \mathrm{~dB}$ in the low frequency range (up to several tens of $\mathrm{MHz}$ ).

In many interferometer systems, both the FM and the AM noise of the laser affect the overall performance of the sensor. The homodyne sensor signal shown in Fig. 5 is measured in the frequency domain. The total interferometer noise reduction is seen to be $23.9 \mathrm{~dB}$ at $280 \mathrm{~Hz}$ compared to the $\mathrm{MH}$ generated noise floor. The frequency component at 1

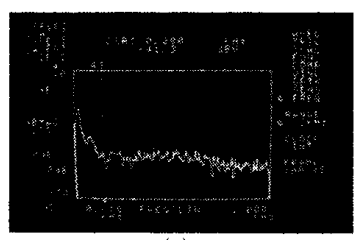

(a)

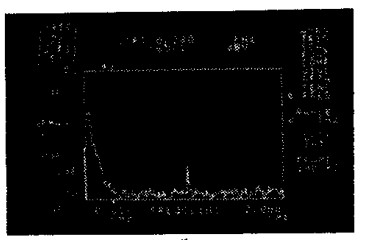

(b)
Fig. 5. The mode hopping noise is degrading an interferometer signal. (a) The output spectrum of a homodyne Michelson interferometer signal when exposed to mode hopping noise from the laser is shown in linear scale, $0-2 \mathrm{kHz}$. (b) Here the excess mode hopping noise is suppressed and the signal to noise ratio is improved by more than $23 \mathrm{~dB}$ compared to (a). The ac modulation is clearly seen in (b).

$\mathrm{kHz}$ seen in Fig. 5(b) is the ac bias signal. The noise reduction is directly related to an improved sensitivity of the sensor. If further noise reduction is needed, quite complex optical stabilization schemes has to be adopted [3]. Using the principles of [3] a 20-25 dB improvement can be obtained but at a price of a considerable complexity and a bulky setup including two separate interferometers in two stabilizing feedback loops.

\section{CONCLuSion}

A simple stabilization scheme for suppression of external cavity mode hopping noise in practical sensor applications was presented. Temperature perturbations, together with optical feedback to the laser cavity, will often degrade the performance of the sensor. To prevent this degradation an all electronic feedback scheme to stabilize the diode laser in a coherent sensor has been demonstrated. The external $\mathrm{MH}$ noise has been suppressed over a wide range of operating currents and optical feedback levels. The stabilizing circuit has been demonstrated to improve the sensitivity of an interferometric sensor by more than $23 \mathrm{~dB}$.

\section{REFERENCES}

[1] R. W. Tkach and A. R. Chraplyvy, "Regimes of feedback effects in 1.5- $\mu \mathrm{m}$ distributed feedback lasers," J. Lightwave Technol, vol. LT-4, pp. 1655-1661, 1986.

[2] M. Ohtsu, Y. Teramachi, Y. Otsuka, and A. Osaki, "Analyses of mode-hopping phenomena in an AlGaAs laser," IEEE J. Quantum Electron., vol. QE-22, pp. 535-543, 1986.

[3] T. P. Newson, F. Farahi, J. D. C. Jones, and D. A. Jackson, "Reduction of semiconductor laser diode phase and amplitude noise in interferometric fiber optic sensors," Appl. Opt., vol. 28, pp. $4210-4215,1989$

[4] P. A. Andrekson and A. Alping, "Electrical mode-hopping noise in external-cavity semiconductor lasers and mode-hopping elimination by a nonoptical control loop," IEEE J. Quantum Electron., vol. QE-23, pp. 2078-2083, 1987.

[5] N. Schunk and K. Petermann, "Numerical analysis of the feedback regimes for a single-mode laser with external feedback," IEEE $J$. Quantum Electron., vol. QE-24, pp. 1242-1247.

[6] M. Osinski and J. Buus, "Linewidth broadening factor in semiconductor lasers-An overview," IEEE J. Quantum Electron., vol. QE-23, pp. 9-29, 1987.

[7] J. H. Osmundsen, "Semiconductor lasers with optical feedback," Ph.D. thesis, LD 55, Electromagn. Inst., Tech. Univ. Denmark, 1985.

[8] C. H. Henry and R. F. Kazarinov, "Instability and semiconductor lasers due to optical feedback from distant reflectors," IEEE $J$. Quantum Electron., vol. QE-22, pp. 294-301, 1986.

[9] S. Piazzolla, P. Spano, and M. Tamburrini, "Characterization of phase noise in semiconductor lasers," Appl. Phys. Lett., vol. 41, pp. 695-696, 1982.

[10] D. Lenstra, B. H. Verbeek, and A. J. den Boef, "Coherence collapse in single-mode semiconductor lasers due to optical feedback," IEEE J. Quantum Electron., vol. QE-21, pp. 674-679, 1985. 